\title{
artigo
}

Campos, G. K. P., Souza, T. C.,Rezende, L. B. M., Rodrigues, A. F. M., Rodrigues, L. A

Representação social da indução do trabalho de parto por gestantes numa maternidade do Espírito Santo

\section{Representação social da indução do trabalho de parto por gestantes numa maternidade do Espírito Santo}

\author{
Social representation of the induction of labor by pregnant women in a maternity in Espírito Santo \\ Representación social de la inducción del parto de mujeres embarazadas en una maternidad en Espírito Santo
}

\begin{abstract}
RESUMO
Objetivo: Avaliar as representações sociais de puérperas acerca da indução do parto. Método: Trata-se de um estudo com abordagem qualitativa realizado com puérperas assistidas em um hospital maternidade de referência no Espírito Santo, no período de junho a novembro de 2021. Para análise dos resultados, as entrevistas gravadas foram transcritas para análises semânticas das informações e extraídas as evocações, elucidadas por meio do software openEVEC 0.92 que permite a formação do núcleo central e o sistema periférico das representações sociais acerca da indução do parto.O estudo foi submetido ao Comitê de Ética em Pesquisa com Seres Humanos do Centro Universitário do Espírito Santo - UNESC.Resultado: As representações sociais referente à indução do parto foram positivas para as puérperas, corroborando com outras pesquisas. Conclusão: É importante que mais pesquisas sejam realizadas a fim de proporcionar o aprimoramento das condutas obstétricas, o que beneficiará o binômio materno-fetal.
\end{abstract}

DESCRITORES: Trabalho de parto induzido; Parto normal;Misoprostol; Índice de apgar.

\section{ABSTRACT}

Objective: To evaluate the social representations of postpartum women about labor induction. Method: This is a study with a qualitative approach carried out with puerperal women assisted at a referencematernity hospital in Espírito Santo, fromJunetoNovember 2021. To analyze the results, the recorded interviews were transcribed for semantic analysis of the information and the evocationswereextracted, elucidatedthroughtheopenEVEC 0.92 software thatallowstheformation of the central nucleus and the peripheral system of the social representations about the induction of childbirth. The study was submitted to the Ethics Committee in Research withHumanBeingsofthe Centro Universitário do Espírito Santo - UNESC. Result: The social representations regarding the induction of labor were positive for the puerperal women, corroborating other studies. Conclusion: It is important that more research is carried out in order to provide the improvement of obstetric procedures, which will benefit the maternal-fetalbinomial.

DESCRIPTORS: Labor, Induced; Natural Childbirth; Misoprostol; Apgar Score

\section{RESUMEN}

Objetivo: Evaluar las representaciones sociales de puérperas sobre la inducción del parto. Método: Este es un estudio con abordaje cualitativo realizado con puérperas atendidas en una maternidad de referencia en Espírito Santo, de junio a noviembre de 2021. Para analizar los resultados, las entrevistas grabadas fueron transcritas para el análisis semántico de las informaciones y las evocaciones. fueron extraídos, dilucidados a través del software openEVEC 0.92 que permite la formación del núcleo central y el sistema periférico de las representaciones sociales sobre la inducción del parto. El estudio fue sometido al Comité de Ética en Investigación con Seres Humanos del Centro Universitário do Espírito Santo UNESC. Resultado: Las representaciones sociales sobre la inducción del parto fueron positivas para las puérperas, corroborando otros estudios. Conclusión: Es importante que se realicen más investigaciones con el fin de brindar la mejora de los procedimientos obstétricos, lo que beneficiará al binomio materno-fetal.

DESCRIPTORES: Trabajo inducido; Parto normal; Misoprostol; Índice de apgar.

RECEBIDO EM: 06/11/2021 APROVADO EM: 17/01/2022

\section{Greice Kelly Palmeira Campos}

Centro Universitário do Espírito Santo - UNESC. Acadêmica de Medicina/ Aluna de Iniciação Científica. ORCID: 0000-0002-5169-5282

\section{Thalita Cossuol de Souza}

Centro Universitário do Espírito Santo - UNESC. Residente de Enfermagem Obstétrica.

ORCID : 0000-0003-0143-4428 


\section{Lívia Bicalho Montebeller Rezende}

Centro Universitário do Espírito Santo - UNESC. Acadêmica de Enfermagem.

ORCID : 0000-0002-3809-1580

\section{Adriene de Freitas Moreno Rodrigues}

Centro Universitário do Espírito Santo - UNESC. ProfessoraUniversitária/ Pesquisadora.

ORCID : 0000-0001-5028-3262

\section{Luciano Antonio Rodrigues}

Centro Universitário do Espírito Santo - UNESC. ProfessorUniversitário/ Pesquisador

ORCID : 0000-0001-5758-456X

\section{INTRODUÇÃO}

0 início do trabalho de parto e parto depende de uma série de processos coordenados e sincronizados, que podem necessitar de ajuda profissional. Em um contexto de respeito aos direitos e desejos das mulheres, da garantia de humanização do parto e nascimento, sobretudobaseado em práticas obstétricas fundamentadas em evidências, a indução do parto é um procedimento que, se bem indicado, pode mudar a realidade, principalmente quantoà realização desnecessária de cesarianas 1,2 .

A indução do parto é um procedimento relativamente comum na prática obstétrica e consiste no desencadeamento artificial de contrações uterinas eficazes antes do início espontâneo do trabalho de parto em gestantes com idade gestacional maior que 22 semanas3. É indicada quando os riscos maternos e ou fetais são maiores do que a manutenção da gestação2.

O Colégio Americano de Ginecologia e Obstetrícia (ACOG) recomenda a indução do parto nos casos de: corioamnionite, morte fetal, hipertensão gestacional, pré-eclâmpsia e/ou eclâmpsia, ruptura das membranas amnióticas, gravidez de $\geq 41$ semanas, certas condições maternas (tais como, diabetes mellitus, doenças renais, hipertensão crônica, entre outros) e comprometimento fetal (por exemplo, restrição de crescimento intrauterino [CIUR], isoimunização e oligodramnia4.

$\mathrm{O}$ método ideal depende das condições do processo de maturação cervical, para evitarmos procedimentos prolongados, exaustivos e com riscos de falha na indução e alterações na contratilidade uterina, elevando-se a incidência de cesárea e aumento na morbidade e mortalidade materna associa$\mathrm{da}$ a fenômenos hemorrágicos, infecciosos e tromboembólicos5,6.

Entre os métodos mais comumente usados para promover a maturação cervical estão à administração de prostaglandinas, sendo o Misoprostol a mais comum, ocitocina e a sonda de Foley, que sozinhas ou em uma combinação ajudam no processo de maturação do colo uterino e estimulam o parto. O método tradicional para avaliar a maturação do colo do útero antes da indução do parto é o sistema de pontuação cervical descrito por Bishop, conhecido como escore de Bishop4,6,7.

Nesse sentido, incorpora-se a promoção da saúde como princípio norteador, com ênfase especial na melhoria da atenção obstétrica, como sugerido pelo Ministério da Saúde no Brasil, no ano de 2004, através da Lei número 10.745/03. Dentre os diversos pontos contemplados pela Lei, está incluída a Política de Atenção Integral à Saúde da Mulher. Um dos pontos observados pelo Programa é o monitoramento dos pactos de redução da taxa de cesarianas em hospitais do SUS e da implementação do Programa de Humanização do Pré-Natal e Nascimento, com o objetivo de resgatar a cultura do processo do parto normal como parto natural8,9.

Considerando o processo de gestar e parir um momento peculiar e que remete diversos sentidos na vida da mulher, espera-se identificar se o uso dos métodos de indução ao parto influencia no desfecho materno e fetal, bem comoas representações sociais apresentadas pelas puérperas submetidas ao processo de indução.

Diante desse pressuposto, o estudo tem como objetivo avaliar as representações sociais de puérperas acerca da indução do parto.

\section{MÉTODOS}

Trata-se de um estudo descritivo transversal, de abordagem qualitativa realizada entre junho e novembro de 2021, com 114 puérperas assistidas em um hospital maternidade de referência no Espírito Santo, que possui salas de parto previamente estruturadas para oferta de indução ao parto.

Foram adotados como critérios de inclusão: gestantes submetidas aos métodos de indução ao trabalho de parto, independente da idade gestacional e idade materna;com indicações obstétricas, tais como pós datismo, diabetes gestacional, síndromes hipertensivas da gestação, ruptura prematura de membranas ovulares (RPMO), comprometimento fetal (por exemplo, crescimento intrauterino restrito [CIUR] eisoimunização); que utilizassem os métodos de indução relacionados à maturação do colo uterino, como: misoprostol, ocitocina e sonda de foley.

Todas as participantes depesquisa foram convidadas para adesão à pesquisa de forma voluntária, assinaram o Termo de Consentimento Livre e Esclarecido (TCLE), conforme determina a Resolução CNS $n^{\circ}$ $466 / 12$ e 510/2016. No caso das participantes menores de idade (12 a 17 anos) ou legalmente incapazes, os responsáveis foram orientados e se coniventes com a pesquisa assinaram o TCLE, a participante de pesquisa também foi convidada a assinar o 


\section{artigo}

Campos, G. K. P., Souza, T. C., Rezende, L. B. M., Rodrigues, A. F. M., Rodrigues, L. A.

Representação social da indução do trabalho de parto por gestantes numa maternidade do Espírito Santo

Termo de Assentimento Livre e Esclarecido (TALE), de acordo com as resoluções supras citadas. Em ambos os documentos as participantes do estudo foram esclarecidas sobre a natureza da pesquisa, seus objetivos, métodos, benefícios previstos, potenciais riscos e o incômodo que esta possa lhes acarretar, na medida de sua compreensão e respeitadas em suas singularidades. Iniciando-se a entrevista após autorização formal por meio da assinatura do TCLE e TALE (caso necessário).

Com o intuito de manter a privacidade, as participantes foram nomeadas de "Puérpera (01), Puérpera (02)”, e assim, sucessivamente, conforme a quantidade de mulheres que aceitaram participar da pesquisa. $\mathrm{E}$ a fim de preservar o caráter espontâneo das falas, os textos não sofreram correções linguísticas/gramaticais.

Foram excluídas gestantes submetidas ao processo de indução que não quiseram participar da pesquisa ou que o responsável legal, para as menores de idade, não autorizasse a sua participação. Além de mulheres submetidas à indução do parto devido óbito fetal.

As entrevistas foram realizadaspor uma das pesquisadoras, no alojamento conjunto do Hospital e Maternidade São José, durante o puerpério imediato das pacientes. Para análise dos resultados, os dados qualitativos foram tratados após a aplicação das entrevistas gravadascom auxílio de um aparelho celular, sendo estas digitalizadas, transcritas na íntegra, para análises semânticas das informações e extraídas as evocações, as quais foram elucidadas por meio do software openEVOC 0.92 , o qual permite a realização de cálculos estatísticos, estabelecendo matrizes de co-ocorrências, que servem de base para a construção do quadro de quatro casas e com base nesse processamento chegamos ao provável núcleo central e sistema periférico da representação social da indução do trabalho de parto.

A teoria das representações sociais (TRS) é uma construção que o sujeito faz para entender o mundo e para se comunicar. O estudo das representações sociais traz em seu escopo algumas preocupações importantes, possibilita compreender como os conhecimentos e valores sobre o outro inferem nas práticas cotidianas 10 .

Já a Teoria do Núcleo Central (TNC), é uma das três formas de se abordar a TRS, sustentada pela hipótese de que toda representação social está organizada em torno de um núcleo central e um sistema periférico. O núcleo central está relacionado à memória coletiva dando significação, consistência e permanência à representação sendo, portanto, estável e resistente a mudanças, enquanto o sistema periférico é responsável pela atualização e contextualização da representação 11,12.

Esta pesquisa foi submetida ao Comitê de Ética em Pesquisa do Centro Universitário do Espírito Santo (UNESC), obtendo o certificado de apresentação para apreciação ética (CAAE) número 46570921.9.0000.5062, e parecer aprovado sob o número 4.719.770, atendendo aos critérios concebidos pela Comissão Nacional de Ética em Pesquisa (CONEP).

\section{RESULTADOS}

No que se refere ao método de indução utilizado,das 114 participantes entrevistadas, $99 \%$ das participantes utilizaram o misoprostol como principal método e apenas $1 \%$ o método de Krause. A quantidade de comprimidos de misoprostol utilizados

\section{Tabela01 - Indicações para a indução do parto.}

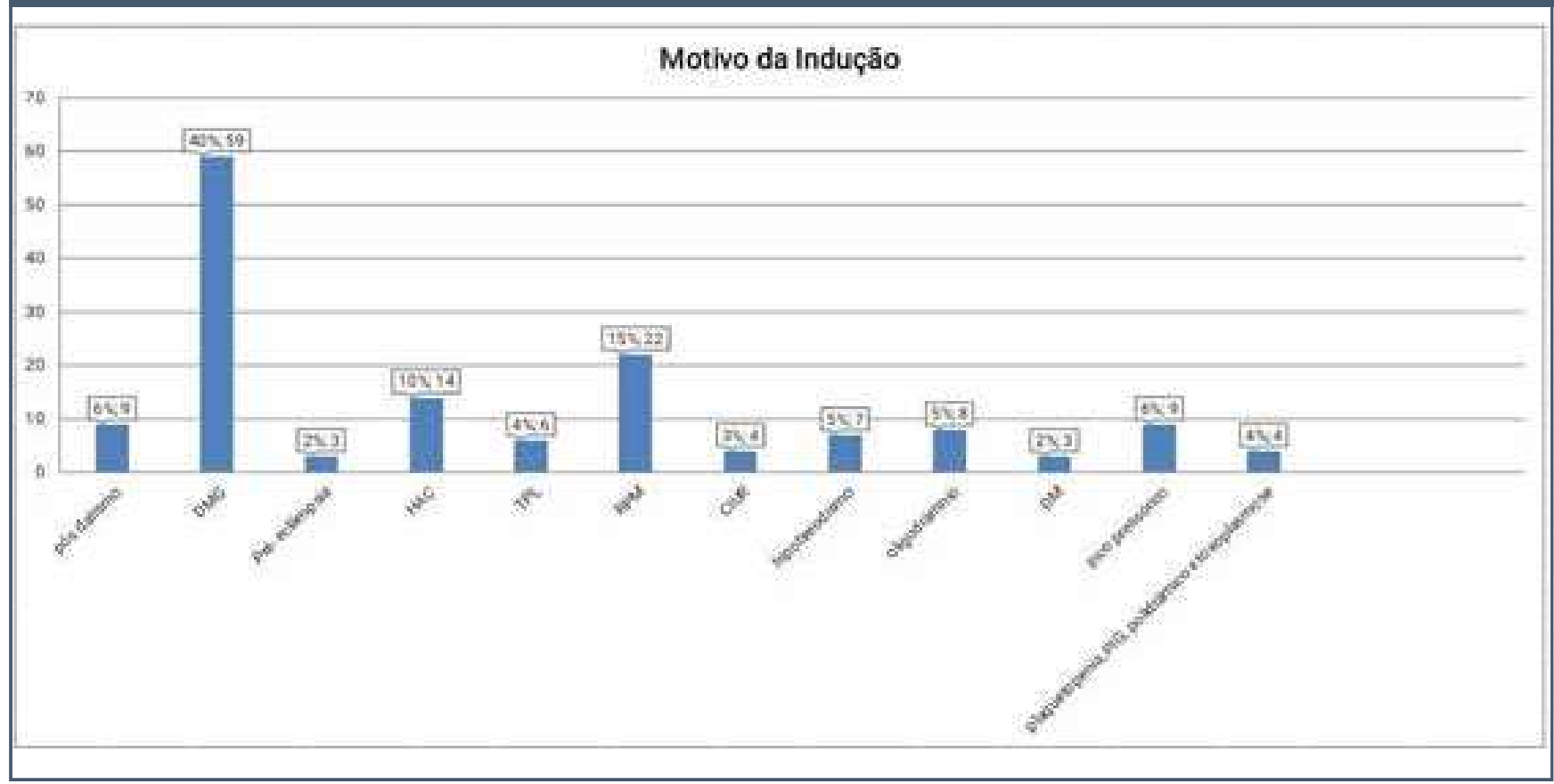

Fonte: Documentos do Hospitale Maternidade São José (HMSJ), 2021 
variou de 1 a 8 comprimidos, distribuídos da seguinte forma: 1 comprimido (18\%), 2 comprimidos (18\%), 3 comprimidos (27\%), 4 comprimidos (13\%), 5 comprimidos (11\%), 6 comprimidos (10\%), 8 comprimidos (2\%). A ocitocina foi utilizada concomitantemente ao misoprostol em $72 \%$ das pacientes.

Os motivos que levaram a indicação da indução foram diversos, como apresentado na tabela 01 , dentre eles: pós-datismo (6\%), Diabetes Melitus Gestacional (DMG) (40\%), Pré-eclâmpsia (2\%), Hipertensão Arterial Crônica(HAC) (10\%), Trabalho de Parto Latente (TPL) (4\%), Rotura Prematura das Membranas (RPM) (15\%), Crescimento Intra Uterino Restrito (CIUR) (3\%), hipotireoidismo (5\%), oligodrâmnio (5\%), DM (2\%), pico pressórico $(6 \%)$ e $4 \%$ representados por plaquetopenia, feto Pequeno para Idade Gestacional (PIG), polidrâmnio e toxoplasmose.

O resultado da associação livre foi uma lista com 199 palavras, sendo que destas 32 (16,08\%) eram diferentes. Observou-se que a palavra "comprimido" apareceu com o maior número de evocações (43), enquanto 16 palavras foram evocadas apenas uma só vez.

Após a organização das evocações pelo softwereopenEVOC originou-se um quadro de quatro casas formado pelos elementos que compõem o núcleo central e a periferia de uma representação, conforme quadro 01 :

\section{DISCUSSÃO}

Durante o delineamento do núcleo central e o sistema periférico das representações sociais acerca da indução do parto por puérperas, através da associação livre de palavras, solicitou-se para as puérperas que relatassem cinco palavras que lhe viessem imediatamente à lembrança ao ouvir a expressão “indução ao parto". Em seguida, pediu-se que elegessem dentre as palavras evocadas a que estimasse ser a mais importante.

A distribuição dos dados das ocorrências em quatro quadrantes nos permite visualizar o núcleo central, os elementos intermediários, de contraste e periféricos da
Quadro01 - Quadro de quatro casas formado pelos elementos que compõem o núcleo central e a periferia de uma representação acerca da "indução ao parto".

\begin{tabular}{|c|c|c|c|c|c|}
\hline \multirow{2}{*}{$\frac{*}{25.6136}$} & \multicolumn{2}{|c|}{ Frequência $\cdots 1.5$ / Ordem de evocaçüo < 1.5} & \multirow{2}{*}{\begin{tabular}{|c|} 
\\
$12.56 \%$ \\
\end{tabular}} & \multicolumn{2}{|c|}{ Frequēncia >in $1.5 /$ Ordem de evocaç̧̄o se 1.5} \\
\hline & comprimiso & 1,4 & & contracao & 1.52 \\
\hline $14.57 \%$ & adiantar o parto & 1,17 & $5.53 \%$ & disatacto & 9.73 \\
\hline $13.57 \%$ & oor & 1,33 & $4,02 \%$ & 3010 & 1,63 \\
\hline 2.015 & medo & 1 & $3.52 \%$ & esperar & 1,57 \\
\hline 2.015 & toque vaginal & 1.25 & $3.02 \%$ & ansiedade & 3.67 \\
\hline 1.515 & nos sabe & 1 & $3.02 \%$ & medo & 2 \\
\hline $1.51 \%$ & demera & 1.33 & 1,518 & samirhar & 2 \\
\hline$\vec{*}$ & \multicolumn{2}{|c|}{ Frequềncia - 1.5 / Ordem de evocaçăo * 1.5} & - & \multicolumn{2}{|c|}{ Frequência $* 1.5$ / Ordem de evocachio $>1.5$} \\
\hline 1.013 & somprimido & 1 & $1.01 \%$ & parto & 15 \\
\hline $0.5 \%$ & cor & 1 & $0.5 \%$ & banno quente & 2 \\
\hline $0.5 \%$ & Camiahar & 1 & $0.5 \%$ & scupuntura & 2 \\
\hline C.SF & supto & 1 & $0.5 \times 6$ & Dota & 2 \\
\hline $0.5 \%$ & ser notural & 1 & $0.5 \%$ & intercorrensis & 2 \\
\hline $0.5 \%$ & amolecer o utero & 1 & $0.5 \%$ & vempo & 2 \\
\hline $0.5 \%$ & iniciar o parto & 1 & $0.5 \%$ & nascimento. & 2 \\
\hline $0.5 \%$ & setificial & 1 & & & \\
\hline $0.5 \%$ & angustis & 1 & & & \\
\hline 0.596 & forces & 1 & & & \\
\hline $0.5 \%$ & ajudar & 1 & & & \\
\hline
\end{tabular}

Fonte: Corpus de análise processado pelo software openEVOC 0.92.

representação. Destarte, para interpretar o quadro, decorremos da seguinte maneira: no quadrante superior esquerdo localizam-se as palavras que constituem, muito provavelmente, o núcleo central da representação, no quadrante superior direito a primeira periferia, no quadrante inferior esquerdo, os elementos de contraste e, por fim, no quadrante inferior direito, a segunda periferia da representação social13.

No primeiro quadrante estão situados os prováveis elementos centrais: "comprimido", "adiantar o parto", "dor", "medo", "toque vaginal”, "não sabe” e "demora”. Já os elementos periféricos da representação social da indução ao parto encontram-se distribuídos nos três demais quadrantes: no superior direito, as palavras "contração" e "dilatação"são os elementosmais evocados pertencentes à primeira periferia; no quadrante inferior esquerdo estão as palavras "comprimido", "dor", "caminhar", "susto", "ser natural", "amolecer o útero", "iniciar o parto", "artificial”, "angústia”, "força” e "ajudar", constituem a zona de contraste, e, no quadrante inferior direito, segunda periferia, estão localizadas as palavras: "parto", "banho quente", "acupuntura", "bola", "intercorrência", "tempo" e "nascimento". Podemos dizer que esses elementos indicam a gama de sentidos atribuídos pelas Puérperas a indução ao parto.

No possível núcleo central a palavra "comprimido" ocupa este quadrante certamente devido ao fato da prática obstétrica no Brasil em inúmeras maternidades se restringir basicamente ao uso de dois fárma$\cos$, a ocitocina e prostaglandinas, em especial a prostaglandina do tipo E1, também conhecida como misoprostol, um comprimido popularmente manifesto 7 .

Essa percepção mostra que apesar de não possuírem conhecimento técnico-científico a respeito do assunto, sabem e reconhecem de maneira generalista a importância da utilização da medicação ("comprimido") para o processo de indução do parto, como afirma uma das participantes:

"Isso aí que eu falei mesmo, adiantar pro parto acontecer. Com comprimido né."

(Puérpera 03) 
"É pra dilatar nascer, parece. Colocando os comprimidos para dilatar." (Puérpera 16)

A palavra "adiantar o parto" segundo elemento do núcleo central, se assemelha ao estudo realizado por Amando e colaboradores (2020) em que as participantesentendem por indução o procedimento que tem como objetivo promover o nascimento antecipado por parto vaginal, diante de suas indicações 14 .

Tais achados corroboram com os dados encontrados na literatura, em que, de maneira mais técnico definem a indução do parto como um método que promove artificialmente o surgimento das contrações uterinas, levando ao trabalho de parto 15 . Também evidenciado pela fala das participantes:

"Acelerar o parto, sentir dor pro parto acontecer."

(Puérpera 08)

"Contrair para dilatar, adiantar o processo do que seria mais natural." (Puérpera 23)

No mesmo raciocínio de interpretação anterior, as palavras "dor" e "medo" podem ser explicados pelo fato de haver estudos que demonstram que o parto induzido apresenta uma fase latente mais longa $e$ muitas vezes dolorosa. Para os pesquisadores, essa percepção pode estar atrelada a vários motivos, como o desconhecimento do processo e as escolhas limitadas de métodos para alívio da dor no trabalho de parto, por exemplo16.

Em relação a palavra "toque vaginal" é de conhecimento que a realização do toque vaginal constitui uma prática corriqueiramente utilizada para avaliar a gestante durante o trabalho de parto. $\mathrm{O}$ exame permite explorar o colo do útero, a bolsa das águas $\mathrm{e}$ a apresentação, além de outras informações importantes. Dessa forma, para a indução de trabalho de parto é necessário a análise das condições citadas no desencadear do processo, visto que, o desígnio e a permanência de determinado método indutor se

fazem as custas dessas informações 17. Os elementos periféricos da representa-

Quanto ao sistema periférico é importante ressaltar que ele é complemento indispensável do central, uma vez

que resguarda esse núcleo, atualiza

e contextualiza

\section{firmemente suas}

deliberações

normativas,

possibilitando

uma diversificação

em detrimento

\section{das experiências}

cotidianas dentre as

quais os indivíduos

\section{estão submersos}

ção social quanto à indução ao parto encontram-se distribuídos nos três demais qua- drantes e os mesmos possibilitam perceber amplos sentidos atribuídos pelas puérperas a essa forma do processo de nascer.

Quanto ao sistema periférico é importante ressaltar que ele é complemento indispensável do central, uma vez que resguarda esse núcleo, atualiza e contextualiza firmemente suas deliberações normativas, possibilitando uma diversificação em detrimento das experiências cotidianas dentre as quais os indivíduos estão submersos. Resumidamente, os elementos do sistema periférico fazem a interconexão entre a realidade tangível e o sistema central. Destarte, pretende-se enfatizar seu valor na configuração das representações sociais acerca da indução ao parto.

No quadrante superior direito situam-se as palavras "contração" e "dilatação" como elementos de maior destaque pertencente à primeira periferia, percebe-se que há conhecimento por parte das participantes quanto aos objetivos da indução, uma vez que a contração e dilatação são respostas esperadas diante de uma indução para o desencadeamento do nascimento, evidenciado pelo American CollegeofObstetriciansandGynecologists (2000), mencionando que o trabalho de parto induzido consiste em desencadear contrações uterinas efetivas antes do deflagrar espontâneo, com o objetivo de promover a dilatação cervical e a descida da apresentação fetal em mulheres com mais de 22 semanas de idade gestacional18.

A palavra "soro",recebeurealcesubsequente. No que diz respeito ao "soro", o uso da ocitocina é o método mais empregado em todo o mundo para indução do parto por diante de sua ação rápida, a menor ocorrência de hiperestimulação uterina e a rápida eliminação após a sua cessação, no entanto está indicada para pacientes com Índice de Bishop maior que 719.

Ainda no núcleo periférico as palavras "esperar" e "ansiedade" corroboram com informações apresentadas por Amando et al. (2020), relatando que a duração do procedimento de indução é complexo e variável, visto que depende de vários aspectos, individuais de cada paciente, como a maturação cervical, escolha do método indutor, 
podendo ser um procedimento demorado. Outra variável que deve ser questionada diz respeito ao local de realização do estudo, uma maternidade referência em alto risco para o noroeste do espírito Santo, que por atender uma grande demanda de pacientes, por vez esse apresenta em condição de superlotação, o que pode influenciar na comunicação entre profissional e paciente, uma vez que o esclarecimento adequado sobre o procedimento requer tempo, assim como a exigência de realização de exames prévios para iniciar a indução, e todos os eventos envolvidos no processo, podem amenizar as dúvidas, os desconfortos, ansiedade e medo com relação à intervenção prestada14.

As palavras "comprimido" e "dor" posicionadas no quadrante inferior esquerdo, de acordo com Oliveira, Marques e Tosoli (2005, p. 4), constituem a zona de contraste, ou seja, ela "comporta elementos que caracterizam variações da representação em função de subgrupos, sobretudo, sem modificar os elementos centrais e a própria representação, ou seja, denotam mudanças ou transição de uma representação social". Assim como receberam maior destaque como elementos centrais, tais palavras se reforçam na zona de contraste13, e podem ser observadas nas seguintes falas:

"É isso mesmo... o comprimido. A dor da contração."

(Puérpera 33)

"A moça falou que ia colocar uns comprimidos pra começar a sentir dor depois."

(Puérpera 44)

No quadrante inferior direito, segunda periferia da representação social quanto à indução ao parto, estão localizadas as palavras: "parto", "banho quente", "acupuntura", "bola", "intercorrência", "tempo" e "nascimento".Para análise agrupamos as palavras "parto" e "nascimento", em seguida "banho quente", "acupuntura" e "bola".

As palavras"parto" e "nascimento",evidenciam pelas participantes que o procedimento de indução tem como objetivo o nascimento antecipado por parto vaginal, quando a continuidade da gestação representa maior risco para a mãe e/ou feto que a sua interrupção. Sob essa perspectiva, o processo de indução tem o papel de funcionar como uma estratégia respeitável e segura para a diminuição dos índices de cesáreas desnecessárias e mal indicadas e, assim, reduzir as possíveis complicações maternas e neonatais advindas do procedimento cirúrgico20. Representado pelas falas a seguir:

\section{"O nascimento do meu filho." \\ (Puérpera 06) \\ "O nascimento da minha filha." \\ (Puérpera 20)}

O "banho quente", "acupuntura" e a "bola”, métodos utilizados na indução do parto enquadrados como estímulos naturais, ou apenas como coadjuvantes do processo, embora não tenha relatos de efeitos teratogênicos conhecidos, apesar da efetividade destas técnicas serem limitadas e não apresentarem estudos randomizados que corroborem estes métodos de indução, no entanto, Vicente et al (2021), relatam que de 100 participantes que utilizaram métodos não farmacológicos para alívio da dor, $79 \%$ evoluíram para parto normal e $29 \%$ para cesariana 21 . Tais métodos são amplamente utilizados e conhecidos pelas participantes, conforme mencionado nas seguintes falas:

\author{
"Ah, ficar andando... usando a \\ bola... banho quente. Me ajudou \\ muito." \\ (Puérpera 37)
}

"São métodos que provocam o parto normal. Como acupuntura, ocitocina, descolamento de bolsa, rompimento de bolsa, os comprimidos que colocaram em mim. Essas coisas..." (Puérpera 68)

\section{CONCLUSÃO}

Inicialmente é necessário salientar quea indução do trabalho de parto é uma tendência da atualidade e importante estratégia para a redução dos seus altos índices de cesarianas. o método mais utilizado para indução do parto foi o misoprostol, garantindo maior taxa de parto vaginal, proporcionando boa vitalidade para o neonato. Oportuno também mencionar que as representações sociais referente à indução do parto foram positivas para as puérperas, assim como a gama de conhecimento prévio a respeito do assunto, identificado na fala das participantes.

Nos resta admitir que os resultados corroboram com outras pesquisas, além disso, alguns dos dados foram comparados com dados internacionais, uma vez há poucos estudos sobre indução de parto e seus desfechos na realidade brasileira. Dessa forma,é importante que mais pesquisas sejam realizadas a fim de proporcionar o aprimoramento das condutas obstétricas, o que beneficiará o binômio materno-fetal.

\section{REFERÊNCIAS}

1. Riscado LC, Jannotti CB, Barbosa RHS. Deciding the route of delivery in Brazil: themes and trends in public health production.Texto Contexto Enferm, v. 25, n. 1, p. 1-10, 2016. Available from: <https:// www.scielo.br/j/tce/a/f9Cr3bm5ySPMNGvWZTr4fVs/?format=pdf\&lang=en $>$.

2. Pfützenreuter GR et al. Fatores associados à cesárea intraparto em mulheres submetidas a indução do parto. Revista brasileira de ginecologia e obstetrícia : revista da Federação Brasileira das Sociedades de Ginecologia e Obstetrícia, v. 41. n. 6, p. 363-370, 2019. Available from: <https://www.thieme-connect.de/products/ejournals/pdf/10.1055/s-0039-1688966.pdf>

3. Silva TAG et al. Indução de parto utilizando misoprostol em um hospital terciário no sudeste do Brasil. Revista brasileira de ginecologia e obstetrícia: Revista da Federação Brasileira das Sociedades de 


\section{artigo}

Campos, G. K. P., Souza, T. C.,Rezende, L. B. M., Rodrigues, A. F. M., Rodrigues, L. A

Representação social da indução do trabalho de parto por gestantes numa maternidade do Espírito Santo

\section{REFERÊNCIAS}

Ginecologia e Obstetrícia, v. 39, n. 10, p. 523-528, 2017. Available from: <https://www.scielo.br/j/rbgo/a/8Z8sjVD96venww6khNC4t$\mathrm{Kn} /$ ?format=pdf\&lang=en>.

4. ACOG. Committee on Practice Bulletins. Obstetrics. ACOG Practice Bulletin No. 107: induction of labor. ObstetGynecol. v.114, n. 2, p.386-397, 2009.

5. Souza GN et al. Métodos de indução do trabalho de parto. Femina, v. 41, n. 1, p. 47-54, 2013. Available from: <http://files.bvs.br/ upload/S/0100-7254/2013/v41n1/a3781.pdf>.

6. Paro HBMS, Catani RR. Indução do trabalho de parto em mulheres com ou sem cesárea anterior: Protocolo Assistencial do Hospital de Clínicas de Uberlândia.Uberlândia:EDUFU, 2019.

7. Almeida LMS, Alexandre RFF, Jesus LKA. Métodos de indução do trabalho de parto: misoprostol, ocitocina e sonda foley, revisão de literatura. Caderno de Graduação - Ciências Biológicas e da SaúdeUnit, v. 4, n. 1, p. 43- 58, 2017. Available from: <https://periodicos.set.edu. br/cadernobiologicas/article/view/3559>.

8. Brasil, Ministério da Saúde. Política nacional de atenção integral à saúde da mulher: princípios e diretrizes / Ministério da Saúde, Secretaria de Atenção à Saúde, Departamento de Ações Programáticas Estratégicas. Brasília, 2004. Disponivel em: <https://bvsms.saude. gov.br/bvs/publicacoes/politica_nac_atencao_mulher.pdf> Acesso em: 25 set. 2021.

9. Ayres LFA, Henriques BD, Amorim WM. A representação cultural de um "parto natural": o ordenamento do corpo grávido em meados do século XX. Ciência \& Saúde Coletiva, v. 23, n. 11, p. 3525- 3534, 2018. Available from: <https://www.scielo.br/j/csc/a/vzyCnXjcGvpzySDMVqVCfXG/?format=pdf>

10. Moscovici S. A Representação Social sobre a Psicanálise. Rio de janeiro: Zahar Editores, 1978.

11. Vèrges P. Conjunto de programas que permitem a análise de evocações: EVOC: manual. Versão 5. Aix en Provence: [S. n.\} 2002.

12. Sant'anna HC. OpenEvoc: um programa de apoio à pesquisa em Representações Sociais. In: Avelar L et al. (Org.). Psicologia Social:desafioscontemporâneos. Vitória: GM Gráfica e Editora, 2012.

13. Oliveira CD, Marques SC, Tosoli AM. Analise das evocações livres: uma técnica de análise estrutural das representações sociais. In: Moreira AP et al. (Org.). Perspectivas teórico-metodológicas em representações sociais. João Pessoa: Ed. da UFPB, 2005.

14. Amando AR et al. Vivência de puérperas no processo de indução do trabalho de parto. Revista Enfermagem Atual In Derme, v. 92, n. 30, p. 156-167, 2020. Available from: <https://revistaenfermagematual.com.br/index.php/revista/article/view/660/635>.

15. Scapin SQ et al. Indução de parto em um hospital universitário: métodos e desfechos. Texto Contexto Enferm, v. 27, n. 1, p. 1-8, 2018. Available from: <https://www.scielo.br/j/tce/a/cCpfS7xth6BTZK5h-

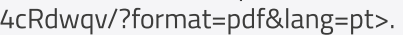

16. Nooh AM, Mohamed ME. Cervical Ripening and Induction of Labor: Awareness, Knowledge, Perception and Attitude of Antenatal Care-Seeking Women at Zagazig University Hospital, Zagazig, Egypt. Open Journal of Obstetrics and Gynecology, v. 5, p. 626-634, 2015. Available from: <https://www.scirp.org/pdf/ OJOG_2015092115420003.pdf>.

17. Montenegro CAB, Filho JR. Rezende Obstetrícia. 13. ed. Rio de Janeiro: Guanabara Koogan, 2017.

18. American College of Obstetricians and Gynecologists. Response to Searle, s drug worning on misoprostol. ACOG committee opinion, n. 248. Washington, D: American College of Obstetricians and Gynecologists; 2000 .

19. Alfirevic Z, Kelly AJ, Dowswell T. Intravenous oxytocin alone for cervical ripening and induction of labour. Cochrane Database of Systematic Reviews. v. 3, 2009. Available from: <https://www.ncbi.nlm. nih.gov/pmc/articles/PMC4164045/>.

20. Weidle WG et al. Escolha da via de parto pela mulher: autonomia ou indução?.Cad. Saúde Colet, v. 22, n. 1, p.46-53, 2014. Available from: <https://www.scielo.br/j/cadsc/a/wRvpVrnwmPcqVLqJTLLcvbb/?format=pdf\&lang=pt>.

21. Vicente, Ingrid G. ., Campos, G. K. P. ., Rodrigues, A. de F. M. ., \& Rodrigues, L. A. . (2020). Representação social dos métodos não-farmacológicos para alívio da dor durante o trabalho de parto. Saúde Coletiva (Barueri), 11(69), 8159-8168. Recuperado de http:// revistas.mpmcomunicacao.com.br/index.php/saudecoletiva/article/ view/1904 